ojs.uv.es/index.php/qfilologia/index

Rebut: 0I.05.202I. Acceptat: 28.09.202I

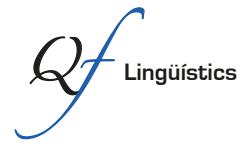

Per a citar aquest article: Pozo-Sánchez, Begoña \& Padilla-Carmona, Carles. 202I. "Criptoginia: una palabra nueva, un concepto para investigar”. Quaderns de Filologia: Estudis Lingüístics XXVI: I75-I92.

doi: $10.7203 /$ QF.26.21983

\title{
Criptoginia: una palabra nueva, un concepto para investigar
}

\section{Cryptogyny: a new word, a concept to research}

\author{
BEGOÑA POZO-SÁNCHEZ \\ Universitat de València \\ begona.pozo@uv.es \\ CARLES PADILLA-CARMONA \\ Universitat de València \\ carles.padilla@uv.es
}

Resumen: La ocultación de los referentes femeninos en los diferentes ámbitos de prestigio social y, por tanto, la desaparición o minusvaloración de las aportaciones de las mujeres en cualquier espacio de poder donde se valore la visibilidad y la notoriedad es un fenómeno muy antiguo al que nadie le había puesto nombre hasta ahora, pero que hemos sufrido sistemáticamente como sociedades. Los autores han creado un concepto que, de forma clara, muestra esta carencia. La palabra criptoginia se compone de dos elementos griegos fácilmente identificables en otras palabras de nuestras lenguas: crypto (ocultar) y gyné (mujer). En este trabajo, además de la argumentación etimológica y lingüística, se presentan una serie de razones culturales y sociológicas que justifican la oportunidad del nuevo término para, finalmente, establecer unas líneas de actuación enmarcadas en la articulación de una propuesta didáctica.

Palabras clave: criptoginia; lenguaje y género; neologismo; violencia(s) de género; educación con perspectiva de género.

Abstract: The concealment of female referents in the different fields of social prestige and, therefore, the disappearance or underestimation of women's contributions to any space of power where visibility and notoriety are valued is a very old phenomenon to which no one had given name so far, but which we have suffered systematically as societies. The authors have created a concept that clearly evidences this shortcoming. The word cryptogyny consists of two Greek elements easily identifiable in other words of our languages: crypto (to hide) and gyné (woman). In this work, in addition to the etymological and linguistic argumentation, the authors present a series of cultural and sociological reasons that justify the opportunity of the new term to, finally, establish lines of action framed in the articulation of a didactic proposal. Keywords: cryptogyny; language and gender; neologism; gender violence(s); education with gender perspective. 


\section{Introducción}

La violencia de género se manifiesta de muy diversas formas: desde aquellas más explícitas, que, desgraciadamente, protagonizan muchos titulares en la prensa y en los informativos -como por ejemplo los asesinatos, las agresiones físicas, incluyendo las violaciones y abusos sexuales-, hasta las más sutiles como el lenguaje y la publicidad sexistas y todo tipo de micromachismos. En medio, todavía, un gran abanico de violencias psicológicas que incluyen las amenazas, los insultos, los gritos, las insinuaciones sexuales fuera de contexto, el desprecio, el chantaje emocional, la culpabilización o la anulación.

Entre aquellas más sutiles, pero no menos lesivas, está la ocultación de la mujer y de cualquier éxito o avance conseguido por una mujer o un grupo de mujeres. Alejando las aportaciones de las mujeres de la vida pública, de los medios de comunicación e incluso de los textos y manuales educativos, se consigue menguar -a menudo anular- el valor de su trabajo y, en consecuencia, su validez como ser humano.

El llamado currículum oculto representa una forma implícita de control social, mediante la cual las clases dominantes intentan a toda costa mantener su poder. Este fenómeno empezó a ser estudiado a principios del siglo pasado; probablemente John Dewey, en su libro Democracy and Education (Dewey, I9I6), fue el primero en llamar la atención sobre esta forma de conformación de la conciencia social con unos fines determinados:

These methods of control are so obvious (because so intentionally employed) that it would hardly be worthwhile to mention them if it were not that notice may now be taken, by way of contrast, of the other more important and permanent mode of control. This other method resides in the ways in which persons, with whom the immature being is associated, use things; the instrumentalities with which they accomplish their own ends. The very existence of the social medium in which an individual lives, moves, and has his being is the standing effective agency of directing his activity (Dewey, I9I6: 33).

El primero en utilizar la expresión currículum oculto (hidden currículum) fue Philip Jackson en i968, mientras que, poco después, Benson Snyder, profesor de psiquiatría en el MIT, desarrolló una teoría completa sobre el concepto (Jackson, I968; Snyder, I97I). Este currículum oculto debe ser desenterrado para revertir el proceso y utilizado en positivo como una herramienta que ayude a la construcción de la identidad de niñas y jóvenes y a su empoderamiento, y, que al mismo tiempo favorezca la educación por la igualdad entre niños 
y niñas para que todos y todas puedan aspirar a las mismas oportunidades futuras.

Pero la ocultación sistemática de los referentes femeninos va mucho más allá de qué se aprende o desaprende en las aulas; alcanza todos los ámbitos de la vida pública, todos aquellos en donde el prestigio social y cualquier parcela de poder están en juego: la política -obviamente-, la historia, la ciencia, la tecnología, el mundo empresarial, y también al arte en todas sus expresiones. Como afirmábamos en un reciente artículo de prensa, en el cual proponíamos por primera vez el término criptogínia (en catalán) para definir este fenómeno,

Aquesta realitat ha provocat la necessitat imperiosa de recuperar les genealogies femenines que han estat sempre amagades a través d'estratègies molt poc innocents: parlem de l'oblit desconsiderat, del fet d'eliminar directament l'autoria de les dones o d'obligar-les a signar amb noms d'home, i, fins i tot, de buscar subterfugis per tal d'imposar autories masculines quan es tracta de descobriments científics. En totes aquestes accions apareix un denominador comú: no reconèixer la vàlua intellectual de les dones $i$, en conseqüència, relegar-les a àmbits on la seua presència no siga -aparentment, és clar!- imprescindible ni visible, això és, l'espai privat de la llar i de les cures -no l'espai públic de la producció i de les relacions de poder (Pozo \& Padilla, 2020).

Las relaciones de poder y su escenificación en el espacio público están precisamente en la base del fenómeno sociológico conocido como efecto Harriet/ Matilda-si bien hoy en día se conoce simplemente como el efecto Matilda- en el ámbito científico. Esta situación de desigualdad fragrante se enmarca en el que fue denominado como efecto Mateo (Martínez Mazaga, 20I4), acuñado en I968 por Robert K. Merton en la revista Science (Merton, I968). Este término proviene de las circunstancias que rodearon el trabajo de tesis de Harriet Zuckerman, a quien el director de su grupo de investigación solo mencionó en las notas a pie de página. Conviene recordar que fue a partir de los estudios para la tesis doctoral de Zuckerman donde la investigadora apuntó las dificultades y el silenciamiento al que se veían sometidos los logros alcanzados por investigadores más jóvenes, siendo atribuidos en cambio de forma generalizada a los miembros que ya ostentaban mayor renombre. El efecto Mateo que ella misma había identificado basándose en la Parábola de los talentos ("Porque al que tiene, le será dado, y tendrá más; y al que no tiene, aun lo que tiene le será quitado", Mateo 25:29), lo padeció al ser despojada del mérito de la investigación. Hubo que esperar veinticinco años hasta que, en i993, la historiadora de la ciencia Margaret W. Rossiter sacara a la luz lo ocurrido en el transcurso 
de la definición del efecto Mateo y pusiera en evidencia las desigualdades de género sobre las que se había construido el discurso científico ${ }^{\mathrm{I}}$. Para nombrar y denunciar públicamente este fenómeno apostó por la duplicidad, identificándolo como efecto Harriet/Matilda en honor a la socióloga que lo había estudiado y, también, a la activista en pro de los derechos de las mujeres, Matilda Joslyn Gage, que fue la primera persona en hacerse eco de este hecho.

En este punto queremos recordar, a modo de ejemplo incontestable, el caso de Lise Meitner (Viena, I878 - Cambridge, I968). Esta científica descubrió el fenómeno de la fisión nuclear, pero fue Otto Hahn, colega suyo en Berlín, quien recibió el premio Nobel por este descubrimiento, obviando la aportación fundamental de su compañera en la investigación. Meitner había podido incorporarse excepcionalmente en la universidad por invitación del profesor Max Planck en una época en la cual estaba prohibido el acceso de las mujeres a las aulas y posteriormente se le "permitió" igualmente investigar en sus laboratorios, pero sin ningún tipo de remuneración. Casos similares encontramos en la historia (no escrita; por tanto, no-historia) de la ciencia, entre los que destacan Nettie Stevens, Marietta Blau, Rosalind Franklin y Jocelyn Bell Burnell, y otras investigadoras que esperamos que vayan emergiendo en el futuro $^{2}$. Entre las pocas excepciones, destaca Marie Curie, el principal referente de las mujeres científicas, que ocupa un lugar destacado en la historia de la ciencia e incluso aparece generalmente en los libros de texto. Su carrera no

\footnotetext{
${ }^{\text {I }}$ Estas desigualdades estaban marcadas especialmente por cuestiones estructurales como las relaciones jerárquicas habituales en los grupos de investigación, el efecto halo de las figuras predominantes o principales y, consecuencia directa de ambas, el silencio sobre las figuras $-\mathrm{y}$ sus aportaciones- que ocupan el plano secundario. El resultado de estas relaciones será un encadenamiento constante de diversas formas de ocultación de los méritos femeninos que, con el término criptoginia, aparecen recogidas en un único concepto.

${ }^{2}$ Si bien en términos generales aún queda camino por recorrer, consideramos importante reconocer la necesidad de acciones específicas y de gran calado social que vayan dirigidas a la visibilización del talento femenino. Es el caso concreto del proyecto Girls4STEM de la Universitat de València, coordinado por la profesora Silvia Rueda Pascual y dirigido al fomento de las vocaciones STEM (Science, Technology, Engineering, Mathematics), muy especialmente entre las jóvenes. Esta colaboración se enmarca en la càtedra de Bretxa Digital de Gènere, creada en octubre de 2020 gracias a un convenio entre la Universitat de València y la Generalitat Valenciana. Las actividades desarrolladas desde estas plataformas tienen como uno de sus objetivos prioritarios presentar y generar referentes femeninos que puedan servir de modelos. Dichos referentes han sido hasta ahora poco visibles, sin embargo, la historia de la ciencia ha recogido en los últimos cuarenta años las aportaciones de las figuras ocultas. Por ejemplo, la historiadora Margaret Rossiter publicó tres tomos (1982, I984, 2012) donde se recoge la labor de las científicas en América.
} 
estuvo exenta de obstáculos, pero sus investigaciones y aportaciones fueron de tal trascendencia que no pudieron dejar de ser tomadas en consideración.

Así las cosas, consideramos que el movimiento decisivo para situarse contra el silenciamiento, es decir, contra la criptoginia, es la ocupación de los espacios, especialmente la del espacio público; puesto que es este el que está vinculado tanto a la visibilidad que conllevan los méritos o la excelencia, como a la idea de autoridad sobre la que se ha asentado el discurso patriarcal con el fin de ocultar las aportaciones y los logros de las mujeres. En las últimas décadas hemos asistido a un proceso de empoderamiento de los discursos producidos por las mujeres, así como a la toma de conciencia sobre la necesidad de la inclusión de la perspectiva de género en todas aquellas cuestiones que les conciernen y que, en general, han sido siempre relegadas a un segundo plano en función de la imposición de límites. Sin embargo, a pesar de estos esfuerzos, la presencia del techo de cristal, de la brecha salarial o la distribución desigual de los cuidados -por nombrar solo tres ejemplos recurrentes en nuestras sociedades- ejercen una violencia sistemática contra las oportunidades y las capacidades a las que se enfrentan las mujeres. Esta tara social, como decíamos, solo puede ser superada con el acceso de la mujer a los espacios públicos, esto es, a los espacios de poder 3 .

En esta perspectiva nos sitúa la académica inglesa Mary Beard cuando sugiere que "lo que necesitamos es cierta sensibilización sobre lo que entendemos por «VOz de autoridad» y cómo hemos llegado a crearla" (Beard, 2018: 5I). Aquí es, pues, inevitable reconducirnos al valor de logocentrismo, al "discurso público acreditado" (Beard, 20I8: I6) que, como manifiesta Beard en su ensayo, ha sido un espacio tradicionalmente negado al discurso de las mujeres y, por lo tanto, ha eliminado así cualquier posibilidad para que se afianzasen como autoridad. En su análisis de la realidad heredada, Mary Beard se muestra clarividente: "en lo relativo a silenciar a las mujeres, la cultura occidental lleva miles de años de práctica" (Beard, 20I8: I2). Si nos centramos en el desconocimiento de la tradición literaria femenina y su repercusión en la falta de autoridad social de las mujeres, Ana López-Navajas y Ángel López

\footnotetext{
${ }^{3}$ La entrevista concedida al periódico Alicante Plaza por la citada profesora Silvia Rueda Pascual pone de manifiesto ya desde el mismo titular ("En el mundo STEM somos pocas y en puestos de responsabilidad casi desaparecemos”) las dificultades a las que se enfrentan las investigadoras en estas disciplinas, tanto por el número reducido de mujeres como por su desaparición en los espacios de decisión. La entrevista completa puede consultarse en el siguiente enlace: https://alicanteplaza.es/silvia-rueda-en-el-mundo-stem-somos-pocas-y-en-puestos-de-responsabilidad-casi-desaparecemos
} 
García-Molins ya mostraban su valoración al respecto, alineándose con las tesis de Beard:

\begin{abstract}
A lo largo del siglo xx se han producido avances hacia la igualdad entre hombres y mujeres en varios ámbitos [...] Sin embargo y a pesar de estos avances, en la actualidad las mujeres aún se encuentran lejos de ver reconocida su autoridad social, es decir, su legitimación social como individuos de pleno derecho y eso se convierte en uno de los más graves impedimentos para el acceso a espacios de poder ya que ese acceso va unido al reconocimiento social. La autoridad social -la auctoritas latina-, lo que entendemos como el saber socialmente reconocido, está unida al ejercicio del poder, la potestas. Potestas que, por lo pronto, no ostentan las mujeres. Esta falta de autoridad social está asentada en el desconocimiento que existe de las contribuciones que a lo largo de la historia han hecho las mujeres a la cultura y al desarrollo humano. Y el desconocimiento de esta tradición de saber femenino es la consecuencia de la práctica exclusión de las mujeres de los referentes sociales que conforman nuestra visión de mundo y nuestra cultura. Esta exclusión, "el hecho de que las mujeres no pasamos a lo escrito", está ya analizada en teoría feminista (Redondo, 200I: 207) y constituye un hecho indiscutible hoy en día. Sobre todo, está ligada a la ausencia de las mujeres que se puede observar en los textos que definen nuestra tradición cultural, entre los que destacan, por su importancia, los manuales de la ESO (López-Navajas \& López García-Molins, 20I2: 27).
\end{abstract}

Este análisis actual de la situación corrobora que la interseccionalidad, acuñada por la teórica estadounidense Kimberlé Crenshaw a finales de los años ochenta y entendida a grandes rasgos como el fenómeno por el cual cada individuo sufre opresión u ostenta privilegio sobre la base de su pertenencia a múltiples categorías sociales, afecta de forma transversal a la mayor parte de los discursos producidos por mujeres. Del mismo modo, otro de los rasgos comunes que presenta el rechazo a la inercia del silenciamiento y la ocultación es, en general, el estudio de estos fenómenos por parte de investigadoras. Precisamente $-\mathrm{o}$, tal vez, desgraciadamente- un sistema habitual cuando se trata de recuperar obras, voces o aportaciones de autoría femenina, como así recuerdan las editoras Júlia Benavent, Elena Moltó y Silvia Fabrizio-Costa en el volumen monográfico dedicado a Las mujeres, la escritura y el poder:

Las voces de las mujeres del pasado, transmitidas por sus escritos, son rescatadas y acompañadas por otras mujeres que, a su vez, además de recuperar del silencio muchos y hermosos testimonios, ofrecen el suyo, suma de su voz y del eco de otras mujeres, en una reflexión también por escrito, plasmada en estos artículos. La propuesta del monográfico se ofreció a todos, hombres y mujeres, y fue secundada por mujeres, excepto en un solo caso. No es una anécdota ese 
singular hecho, como tampoco lo es que los artículos aquí reunidos sean un mapa en el espacio y en el tiempo de una realidad hecha a pinceladas, tan finas, de trazo tan delicado, que apenas se percibe y se conoce. No son, ya lo sabemos todos y todas, los trazos seguros y duros de un tatuaje en un brazo rudo y curtido, sino la tenue y transparente piel de quien se expone poco al sol, de quien se recoge a menudo entre las paredes silenciosas de la casa. Con todo, la voz es clara y firme. Tiene el timbre nítido, expresa un mensaje inequívoco, a lo largo de los siglos y, contra todos los obstáculos, de forma constante, ininterrumpida (Benavent, Moltó \& Fabrizio-Costa, 20I2: 9-IO).

Así pues, esta cadena constante supone una labor tanto de reconocimiento como de recuperación que atraviesa previamente el territorio del desconocimiento y del ocultamiento intencionado -como ha demostrado la académica, escritora y feminista Joanna Russ en su conocida obra Cómo acabar con la escritura de las mujeres (20I9). Es nuestro deber intelectual, como profesores implicados en desenmascarar estos fenómenos y, por lo tanto, en identificar y nombrar la criptoginia, apostar tanto por la recuperación de las voces silenciadas como por la necesidad de generar nuevos espacios donde puedan mostrar todo su talento.

La investigación de Ana López-Navajas (2015) supone un cambio de perspectiva fundamental, además de una inflexión en el estudio de la situación en España -claramente aplicable a otras tradiciones- sobre la transmisión cultural, que califica de amputada y empobrecida, al situar las aportaciones de las mujeres en un espacio irrelevante, lo que supone una constante minusvaloración social que las condiciona negativamente y que las convierte en ciudadanas de segunda. Esta historia sesgada da lugar a una herencia cultural fallida que, como sostiene López-Navajas, excluye sistemáticamente de sus contenidos las aportaciones culturales y científicas de las mujeres en todos los ámbitos. La identificación de la criptoginia en las diversas áreas de conocimiento será clave para poder subvertir, por lo tanto, esta herencia y abogar por una educación plural e igualitaria que cuestione los estereotipos de género que, hoy en día, se siguen perpetuando en nuestras sociedades.

\section{Cuando nombrar se convierte en un acto político}

Una sociedad la mitad de la cual asesina, maltrata, margina, menosprecia o ignora a la otra mitad es una sociedad enferma. Igualmente, si aquella mitad, o su mayoría, no es consciente de este hecho o, siéndolo, no hace nada para solucionarlo. El conjunto de la sociedad debe reconocer la enfermedad, 
identificar el problema, para después intentar poner remedio. Initium est salutis notitia peccati ("el conocimiento del error es el inicio de la curación") nos recordaba Séneca en una de sus cartas a Lucilio4; en términos médicos, se necesita un diagnóstico preciso antes de iniciar cualquier tratamiento. Pero el diagnóstico es claro: durante siglos y en la mayoría de las culturas, en el ámbito de una sociedad machista y patriarcal, se ha producido y se siguen produciendo una serie de violencias de género, como las que apuntábamos anteriormente; entre ellas, una silenciosa y sutil, pero muy nociva: la ocultación sistemática e interesada de los logros conseguidos por mujeres y de aquellas que los han alcanzado, con la intención de no reconocer su validez intelectual y relegarlas a los ámbitos menos relevantes o visibles y alejados de todo prestigio social. Así hemos asistido a la perpetración de una violencia simbólica continuada en el tiempo que, si bien era reconocida, no era nombrada y, en consecuencia, quedaba en una zona de sombra difícilmente identificable.

Sin embargo, una vez identificado el problema, el siguiente paso era ponerle un nombre, darle una sustantividad, ya que la conciencia social se conforma con las acciones y omisiones, con los silencios y con las palabras: lo que no se incluye en los diccionarios aparentemente no existe. Por esta razón propusimos recientemente un neologismo que evidenciara esta carencia y que fuera muy fácilmente reconocible, a la vez que exportable o traducible a otras lenguas. El latín y el griego, con su flexibilidad y su capacidad de crear derivados y compuestos de manera recursiva, son fuente inagotable para la formación de nuevos términos comprensibles para cualquier hablante de una lengua de origen indoeuropeo, como son casi la totalidad de las lenguas occidentales. El término criptoginia se compone de dos lexemas griegos que aparecen también en otras palabras de nuestras lenguas más próximas: crypto (esconder, ocultar) y gyné (mujer)5. Por extensión, el adjetivo derivado debería ser criptógino/a: "dicho de una persona que ejerce la criptoginia" o también "perteneciente o relativo a la criptoginia o a la persona que la ejerce". En catalán, nuestra primera propuesta, que ha tenido un rápido y extenso recorrido,

\footnotetext{
${ }^{4}$ Epístolas morales a Lucilio, 28-9. Aunque el propio Séneca reconoce que "cree que la frase la pronunció Epicuro": mihi hoc dixisse videtur Epicurus (íbidem).

5 Véase, por ejemplo, criptología (estudio de los sistemas, claves y lenguajes ocultos y secretos), criptorquidia (descenso de uno o ambos testículos hasta ocultarse en el escroto), criptomoneda (moneda digital encriptada para evitar falsificaciones), ginecología (rama de la medicina especializada en el aparato reproductor femenino), misoginia (aversión a las mujeres o falta de confianza en ellas), etc.
} 
como veremos a continuación, es criptogínia. El adjetivo derivado, por lo tanto, debe ser criptogin / criptògina ${ }^{6}$.

Este neologismo tiene, como hemos indicado anteriormente, un origen muy claro además de fácilmente identificable; sin embargo, a pesar de estas características, no existía hasta que lo creamos en febrero de 2020 . Todavía ahora nos parece incomprensible que un fenómeno que ha cercenado tantas oportunidades, tantas posibilidades, tantos futuros careciese de un término lingüístico concreto. Por ello consideramos imprescindible nombrar esa realidad y, para ello, pusimos en marcha tres acciones -creer, crear y crecer- que se revelaban necesarias para contrarrestar los límites del lenguaje -a la manera wittgensteiniana- a los que habíamos sido sometidos hasta ese momento. La necesidad de nombrar (creer), de inaugurar (crear) una nueva mirada sobre el mundo y de focalizar, a través de dicha mirada, ese agujero negro de la historia que se había tragado las aportaciones de tantas y tantas mujeres era la única forma posible de recuperarlas (crecer). De esta forma, fusionando las potencialidades de estos tres verbos acuñamos un neologismo que, por fin, impidiese el ocultamiento, durante otros tantos siglos, de las violencias ejercidas sobre las mujeres7. Como en casi todo, tampoco aquí fue casual que estos tres infinitivos se convirtiesen en el humus necesario para la creación lingüística, ya que los tres compartían la misma raíz indoeuropea que el primer componente de la palabra criptoginia: *kar-/kr- $(* \text { ker- } / * \text { kor- })^{8}$. Si nos remontamos al indoeuropeo más antiguo, podemos identificar que la raíz básica *kar-/ kr-, junto con sus variantes *ker- y *kor-, tenía tanto un valor nominal, que hacía referencia a las "piedras u objetos duros" (griego kraníon "casco", ceres "<grano de> trigo"), como un valor verbal, que implicaba otros significados asociados del tipo "moler, desmenuzar, crear" (latín cerno "triturar"> "discernir", "entender"; cresco "crecer"). Y en esta encrucijada etimológica observamos cómo la belleza del origen nos regala, cuando buceamos en el lenguaje, magníficas conexiones que nos alumbran y nos indican el camino, a veces demasiado ensombrecido por tanto ruido. Nuestra voluntad entonces fue la de nombrar para constituir una nueva realidad; porque ampliar los límites del lenguaje y ensanchar el conocimiento del mundo responde también a las

\footnotetext{
${ }^{6}$ Existe una variada bibliografía sobre la creación y el uso de los neologismos. Por aportar solo un par de referencias del mismo autor, véase Alvar Ezquerra (I993; I999).

7 Sobre la necesidad de neologismos para una revolución, véase Mercier (2009).

${ }^{8}$ Las raíces indoeuropeas básicas tienen la estructura CVC (consonante-vocal-consonante); la vocal básica suele ser la /a/, que, dependiendo del contexto fónico, sobre todo al ampliarse la raíz mediante sufijos, puede desaparecer o cambiar su timbre en /e/ o en /o/.
} 
enseñanzas feministas que apostaban por la ocupación del espacio público. Si "lo personal es político" -como se divulgó en la segunda ola del feminismo gracias al ensayo de Carol Hanisch, Lo personal es político, publicado en I970también el hecho de nombrarlo debe serlo.

Como hemos indicado anteriormente, a partir de nuestra propuesta (criptogínia) la palabra comenzó su andadura en congresos, publicaciones, medios de comunicación y redes sociales. Con el fin de atestiguar las reacciones iniciales al término, citaremos aquí tan solo las primeras menciones a este: por ejemplo, el primer retuit del concepto lo llevó a cabo el mismo día en que se publicó el artículo, el 5 de febrero de 2020, Elena Martínez, profesora de derecho y vicerrectora de Igualdad, Diversidad y Sostenibilidad de la Universitat de València; la primera conferencia que se dictó con la incorporación de dicho vocablo fue la de la propia profesora Pozo en las Jornadas "Oficio de los días", organizadas en Córdoba por la Fundación Manuel Álvarez Ortega en marzo de 2020. Su intervención llevaba por título: "Crear, crecer y creer: estrategias necesarias contra la criptoginia"; la primera referencia en prensa escrita apareció en septiembre de 2020, en un artículo del profesor Francesc Martínez Gallego, donde apuntaba:

Esdevé sovint que una realitat, de tan assentada, sembla invisible, com la carta del famós conte d'Edgar Allan Poe: tots la tenien davant dels nassos i no la veien. Criptogínia és l'ocultació dels referents femenins en qualsevol àmbit, des de la literatura a la política, des de la filosofia a la ciència de la naturalesa. Com ha demostrat en els seus interessantíssims estudis Ana López-Navajas, amagar la realitat de les dones suposa convertir els manuals d'ensenyament actual en un seguici d'homes benefactors de la humanitat, mentre elles ocupen un paper secundari. Són menystingudes. Criptogínia hauria d'entrar en el diccionari i, més encara, hauria de normalitzar-se. Millor dit, hauria de ser batuda com a paraula per una nova realitat. Però, mentrestant, deixeu-la passar.

Poco después, apareció un artículo del profesor Rafael Roca (2020) con motivo del 25 de noviembre, Día internacional para la eliminación de la violencia contra las mujeres, donde se ponía de manifiesto cómo el nuevo término podía trasladarse perfectamente a la Valencia del barroco, cuando:

Cinc segles enrere, una bona part dels hòmens sotmetien i maltractaven les dones; i també, com amagaven i/o manipulaven -en benefici propi, és clar-les seues aportacions socials. És a dir, com practicaven l'ocultació de les dones o «criptogínia», «una paraula nova per a un fenomen antic», tal com han teoritzat els amics Carles Padilla i Begonya Pozo. Un exercici que, dissortadament, 
continua viu entre nosaltres, i que hauríem d'afanyar-nos a eradicar d'una vegada per sempre.

Afortunadamente para nuestro neologismo, la sugerencia del profesor Martínez Gallego no pasó desapercibida. La Acadèmia Valenciana de la Llengua (AVL) decidió incorporarla a su Diccionari Normatiu Valencià (DNV) en el pleno del 27 de noviembre de 2020; así que, ocho meses después de su nacimiento, el término criptogínia ${ }^{9}$ obtenía carta de naturaleza y era reconocida por la comunidad científica. A partir de este momento aparecieron diversos artículos donde no solo se celebraba el mérito de la invención léxica, así como el logro de esta rápida incorporación, sino que también se hacía referencia a la necesidad de su extensión e incorporación en uno de los símbolos lexicográficos por excelencia del mundo catalanohablante: el diccionario del Institut d'Estudis Catalans (IEC). Así la escritora Núria Cadenes (2020) concluía un artículo considerando que: "Ara només cal, per acabar de reblar el clau, que el $I E C$ segueixi l'exemple de l'AVL i, posant en pràctica i fent valer les fluides relacions que amb naturalitat mantenen, inclogui el mot al seu, de diccionari. Al seu que, al capdavall, és el de tots". Su deseo de ver incorporado el término al diccionario común de todos los hablantes de la lengua catalana es similar al que manifiesta la académica Amparo Zacarés cuando, con motivo de la inclusión de nuestro neologismo en el $D N V$ por parte de la $A V L$, reflexiona sobre la necesidad de su incorporación -ciertamente urgente- en el diccionario por antonomasia del español, el de la RAE. Opina la profesora Zacarés que:

El diccionario académico y lexicográfico es el libro por excelencia, ya que contiene las palabras con las que nombramos el mundo por orden alfabético. En él solo aparecen aquellas voces que han recibido la autorización de la institución que se encarga de ello como sucede, por ejemplo, con la Real Academia de la Lengua Española. Pero ésta, fiel a su lema de «limpia, fija y da esplendor», sigue ignorando la importancia de analizar la visión patriarcal que representa el lenguaje. De tal modo que resulta urgente detectar las lagunas léxicas y las ausencias temáticas que existen en el diccionario de la RAE. Y no me refiero solo a los rasgos de desigualdad de género con los que el lenguaje utiliza palabras de significado peyorativo para aludir a las mujeres. De hecho, es de sobra conocido que muchas de las expresiones son declaradamente machistas y que responden a atavismos y pautas culturales misóginas muy arraigadas. En lo que en realidad quiero centrarme, es en la necesidad de acuñar nuevos términos que den cuenta de una sociedad paritaria y nombren a las mujeres

9 https://www.avl.gva.es/lexicval/ 
en la medida que desempeñan profesiones diversas y participan de actividades que antes quedaban reservadas a los hombres. Por ahora, la RAE no incluye 'criptoginia' en su diccionario y sería de agradecer que lo hiciera pronto por aquello de llegar a tiempo y no perder el tren de la igualdad. Desde luego, María Moliner, autora del Diccionario del Uso del Español (DUE), lo hubiera hecho.

La urgencia reclamada por la profesora Zacarés para el diccionario de la RAE sí que ha sido tenida en cuenta, en cambio, por el diccionario bilingüe más consultado de la lengua vasca, el diccionario Elhuyar. ${ }^{\mathrm{IO}}$ El 8 de marzo de 202I fue incluida en el diccionario el término kriptoginia, así como su traducción y definición en español: "criptoginia: ocultación de referentes femeninos". Un día después, el 9 de marzo de 202I, otro importante diccionario bilingüe, Labayru, también incorporaba ambos términos. ${ }^{\text {II }}$ En otras lenguas próximas, como el francés, aunque el término no ha sido incorporado oficialmente a un diccionario, ya ha sido empleado por docentes e investigadoras de la Université Paris 8 en el seminario titulado De la cryptogynie à la médiatisation des écrivaines: nouvelles aproches de la littérature catalane, que tuvo lugar entre los meses de febrero y abril de 202I. ${ }^{\text {22 }}$

Tras las repercusiones en prensa, de las que solo hemos presentado una pequeña muestra, y la incorporación del término en algunos diccionarios, encontramos por fin una referencia en una publicación científica en el prólogo de Vicent Josep Escartí y Rafael Roca (eds.) del monográfico En los márgenes de la historia: marginales y minorías:

Recientemente, la Acadèmia Valenciana de la Llengua ha incluido, en su Diccionari Normatiu Valencià, el cultismo "criptogínia", un término creado y difundido desde la Facultat de Filologia, Traducció i Comunicació de la Universitat de València, que la entidad normativa no ha dudado en asumir y definir como "ocultación de los referentes femeninos". Al respecto, resulta evidente que la elaboración del neologismo es fruto de una nueva sensibilidad; más integradora e igualitaria que, con el inicio del siglo xxi, ha empezado a manifestarse entre sectores académicos y sociales. Una sensibilidad más tolerante y

\footnotetext{
1o https://hiztegiak.elhuyar.eus/eu/kriptoginia

${ }^{\text {II }}$ https://hiztegia.labayru.eus/emaitza/LH/all/kriptoginia/256I375?locale=eu

I2 https://la-creatis.univ-tlse2.fr/accueil/manifestations-scientifiques/de-la-cryptogynie-a-la -mediatisation-des-ecrivaines-nouvelles-approches-de-la-litterature-catalane Véase también: https://etudes-romanes.univ-paris8.fr/?2020-202I-Seminaire-interuniversitaire-dematerialise-De-la-cryptogynie-a-la donde se incluyen los videos del seminario, además del programa o el perfil «Cryptogynie» de Youtube, creado para la ocasión: https://www.youtube.com/channel/ UCTu5BLZG2vLNZZBoG_irkzA
} 
plural que fija la mirada en los márgenes sociales, en los personajes históricos obviados y/o rechazados por los discursos oficiales (Escartí \& Roca, 202I: 7).

La recepción mediática y académica del neologismo ha sido, hasta ahora, muy interesante. Especialmente porque el término ha sido empleado tanto por personas expertas en lexicografía como por hablantes de las distintas lenguas mencionadas a través, especialmente, de las redes sociales, que cada día aumentan su influencia en los cambios lingüísticos y en la opinión pública. En todos los ejemplos mencionados se pone en evidencia, por una parte, la necesidad de nombrar como acto político, puesto que supone la constatación irrefutable de un fenómeno social que ha lastrado el trabajo meritorio de las mujeres; por otra parte, la satisfacción que manifiestan las y los hablantes por haber encontrado un término que identifica una situación injusta-ya identificada y descrita en su momento por el efecto Matilda y que ahora el término criptoginia recoge en todas sus diferentes variantes-, denunciable y, sobre todo, que debería ser corregida lo antes posible. Para ello será fundamental la implicación no solo de las personas que nos dedicamos a la filología y la investigación en el ámbito académico, sino también, y muy especialmente, de las que desde el aula trasmiten contenidos curriculares -obviamente condicionados por los materiales que proporcionan las editoriales, a las que habrá que exigirles, mediante los mecanismos legalmente establecidos desde las instancias políticas pertinentes, un mayor compromiso con la igualdad a la hora de revisar y actualizar los contenidos de los libros de texto. El compromiso con una sociedad justa e igualitaria dependerá, pues, de todas las instancias implicadas en el proceso educativo.

\section{Propuesta didáctica}

Las cuestiones apenas mencionadas implican retos diferentes según los distintos niveles educativos. En nuestro caso, centraremos la propuesta didáctica en el ámbito universitario, si bien consideramos que, a modo de cascada, los cambios generados en la educación superior deberían permear otras estructuras y, muy especialmente, al alumnado que después será el responsable de impartir contenidos, así como de trasmitir valores y actitudes en los otros niveles de enseñanza. Este cambio sabemos que no será inmediato, pero desde las aulas universitarias deberíamos ser capaces de garantizar un trasvase de contenidos que conlleve una modificación sustancial de estos, centrada en la incorporación sistemática de la perspectiva de género. Dicha transformación 
implica, por un lado, el avance indiscutible en el marco de la investigación; por otro, la capacidad para hacer llegar, desde la esfera universitaria -mediante un diálogo fluido a través de grupos de trabajo transversales e interdisciplinares-al resto de actores presentes en el proceso educativo -clase política, tejido editorial, espacios educativos-, la necesidad de equilibrar los contenidos a fin de trasladar al alumnado una visión más completa y justa de las aportaciones de las mujeres en todas las disciplinas. Para poder contrarrestar los efectos que ha producido la criptoginia a lo largo de los siglos no bastará solo con la búsqueda y la reparación de los referentes femeninos en el ámbito de la investigación, sino que será necesario articular mecanismos que garanticen posteriormente su presencia en el currículo escolar y, en nuestro caso particular, en las guías docentes. Solamente cuando la concienciación llegue a los más jóvenes, será posible que comiencen a producirse bottom up los cambios en la sociedad de manera natural.

Según nuestro actual sistema educativo, las guías docentes tienen un valor contractual con el alumnado y en estas se recogen todos los aspectos considerados imprescindibles para garantizar su formación en cada módulo o asignatura. Sin embargo, desde nuestra experiencia docente, sabemos que, si bien desde una institución universitaria como la Universitat de València se dedican numerosos recursos a la formación del PDI en perspectiva de género -tanto en docencia como en investigación, como puede comprobarse en la web de la Unitat d'Igualtat-, no siempre se produce un cambio efectivo en los programas de las asignaturas. Esta cuestión debería analizarse en profundidad, tal y como está previsto en la acción 27 del III Pla d'Igualtat 20192022 -dedicada a generar un sistema de medición para monitorizar el grado de incorporación de la perspectiva de género en las guías docentes, es decir, que dé cuenta de la inclusión de referentes femeninos en los temarios y la bibliografía, además de revisar el uso del lenguaje inclusivo-; si no medimos con exactitud este parámetro, difícilmente podremos intervenir y buscar soluciones que reviertan la inercia a la que, en muchas ocasiones, se ve sometido el profesorado que se enfrenta -por diversos motivos- a la falta de tiempo a la hora de poder abordar con profundidad los cambios que sería necesario incorporar en los temarios para introducir en estos, de forma rigurosa, la perspectiva de género. Este aspecto resulta clave, puesto que la modificación de los contenidos, ligada a la formación y la investigación, repercute de forma directa en la transmisión de estos. El esfuerzo por desterrar la criptoginia de nuestras aulas pasa, en primer lugar y necesariamente, por la capacidad investigadora del sistema universitario para, en segundo lugar, trasladar sus 
logros tanto a las guías docentes como a las actividades complementarias en las que el alumnado compruebe los méritos de las mujeres y sus aportaciones en los distintos ámbitos. Para ello también será fundamental reconocer el esfuerzo del profesorado a través de mecanismos que valoren positivamente su dedicación, como puede ser el reconocimiento de méritos específicos ligados a la incorporación de la perspectiva de género en las asignaturas impartidas tanto en las acreditaciones de AVAP-ANECA como en la evaluación del DOCENTIA.

En este punto conviene recordar que la Universitat de València creó en 2014 el Premio A. Olga Quiñones Fernández, con la finalidad de "promoure la incorporació de la perspectiva de gènere i la igualtat en la docència i en la investigació" a través del reconocimiento de la excelencia en trabajos de fin de grado, trabajos de fin de máster y tesis doctorales que aborden cuestiones relacionadas con todas las áreas de investigación. También la Cátedra de Cooperación al Desarrollo Sostenible de la UV premia cada año a los mejores trabajos académicos centrados en el eje de "Personas" de los Objetivos de Desarrollo Sostenible de la Agenda 2030 de Naciones Unidas. Desde nuestro punto de vista, premiar TFG, TFM y tesis con perspectiva de género es una labor magnífica para fortalecer el progreso y el cambio, ya que reconoce el esfuerzo del alumnado y del profesorado que tutoriza y dirige estas investigaciones. Sin embargo, consideramos que cabría realizar un ulterior esfuerzo para conseguir que todo el alumnado de cada centro asistiese a unas Jornadas de Formación e Investigación en Género donde, de primera mano, tuviese la oportunidad de conocer las aportaciones recientes que realiza el profesorado en estas cuestiones. Para ello sería necesario que dichas jornadas se desarrollasen, especialmente, antes de la asignación de tutora o tutor del TFG y que fuesen organizadas desde los decanatos, con la voluntad de proponerlas como actividad de centro y, en consecuencia, de garantizar la presencia de todas las áreas de estudio. Dentro de dichas Jornadas también sería deseable que participara alumnado de cursos anteriores que, siguiendo la filosofía del exitoso programa Entreiguals (mentoria d'estudiants) de la Universitat de València, orientase también al alumnado con sus aportaciones en los estudios de género. En el caso del TFM, al ser un escalón más en el ámbito de la investigación, el alumnado tendrá ya referencias sobre las cuestiones relacionadas con el género y la investigación al haber abordado la redacción de un TFG previo. Esta situación se dará también en el alumnado que decida ampliar sus estudios de doctorado. En este punto queremos matizar que, de la misma manera que la incorporación de la perspectiva de género y la revisión de materiales es 
fundamental en la ESO, como hemos indicado, por tratarse de la enseñanza obligatoria que garantiza la formación en igualdad para todas las personas, en el ámbito universitario esta cuestión debería garantizarse en el grado -ya que no todos los egresados y egresadas continúan posteriormente estudios de máster y doctorado.

El tercer paso que se debería llevar a cabo, según nuestra propuesta didáctica, sería la conformación de grupos de trabajo donde profesorado universitario y de enseñanza primaria y secundaria creara contenidos específicos que garantizasen la visibilidad de las aportaciones de las mujeres ${ }^{13}$. Dichos contenidos podrían estar disponibles para todo el profesorado en un banco de datos; si bien sería deseable que, desde las instancias políticas -consejerías y ministerios implicados- se tomasen las medidas oportunas para revisar los contenidos de los libros de texto y se buscasen estrategias que pusiesen en valor la actualización de los contenidos desde la perspectiva de género como, por ejemplo, aumentar las cuantías de las ayudas -directas o indirectas, según las distintas comunidades autónomas- a aquellas editoriales que se implicasen de forma activa en la revisión de sus contenidos para promover el cambio social y cultural que supondrá el destierro de la criptoginia de los materiales y de las aulas.

La historia de desigualdades a la que nos hemos enfrentado como profesionales de la investigación y la enseñanza nos ha llevado a la creación de un término que identifica de forma clara este fenómeno injusto y que apuesta por su eliminación. En este momento consideramos imprescindible el paso del ámbito teórico al práctico. Por ello hemos formulado la articulación de jornadas educativas que pongan en primer plano las aportaciones de las mujeres en las diferentes disciplinas y que muestren a nuestro alumnado las posibilidades de avance cultural y social que se abren una vez que introducimos la perspectiva de género en la investigación. En estas jornadas se pueden organizar también grupos de debate sobre temarios de las diferentes asignaturas, entre alumnado, para su valoración y discusión con el profesorado.

\footnotetext{
${ }^{13}$ En este punto es importante mencionar la existencia del proyecto Women's Legacy: our cultural heritage to equity, coordinado por la investigadora Ana López-Navajas y liderado por la Conselleria d'Educació de la Generalitat Valenciana. Como aparece indicado someramente en la página inicial, se trata de un "Proyecto europeo que contribuirá de manera decisiva a la inclusión de las mujeres protagonistas de la historia y la cultura y su legado en los contenidos educativos". Puede consultarse el estado de las investigaciones e información sobre este en el siguiente enlace: https://womenslegacyproject.eu/ca/home/
} 
Si la Universidad quiere seguir siendo, como hasta ahora, un factor decisivo del cambio social y no quiere perder la oportunidad de liderar la transformación cultural deberá redoblar sus esfuerzos en todos los aspectos que la constituyen -científico, académico, docente y de gestión- con la finalidad de instituir las bases para un avance social que no renuncie a los parámetros de justicia e igualdad. Sin estos, no podrá ser considerada como la universidad que necesita la sociedad del siglo xxi.

\section{Bibliografía}

Alvar Ezquerra, Manuel. I993. La formación de palabras en español. Madrid: Arco Libros.

Alvar Ezquerra, Manuel. 1999. El neologismo: caracterización, formación y aceptabilidad". En González, J. M.; Montero, M. L. \& Terrón, J. (ed.) V Jornadas de metodología y didáctica de la lengua española: el neologismo. Cáceres: Universidad de Extremadura, 39-66.

Beard, Mary. 20I8. Mujeres y Poder: un manifiesto. Barcelona: Crítica.

Benavent, Julia; Moltó, Elena \& Fabrizio-Costa, Silvia. 20I2. Las mujeres, la escritura y el poder. Quaderns de Filologia: Estudis Literaris I7, 9-II.

Cadenes, Núria. 2020. Criptogínia, el nom de la cosa. Vilaweb.cat. https://www. vilaweb.cat/noticies/criptoginia-el-nom-de-la-cosa/

Crenshaw, Kimberlé. 20ı6. On Intersectionality: Essential Writings of Kimberlé Crenshaw. New York: The New Press.

Dewey, John. I9i6. Democracy and Education. New York: The MacMillan Company.

Escartí, Vicent Josep \& Roca, Rafael (ed.) 202I. En los márgenes de la historia: marginales y minorías. Valencia: Pórtico.

Jackson, Philip Wesley. I968. Life in Classrooms. New York: Holt, Rinehart and Winston.

López-Navajas, Ana \& López García-Molins, Ángel. 20I2. El desconocimiento de la tradición literaria femenina y su repercusión en la falta de autoridad social de las mujeres. Quaderns de Filologia - Estudis Literaris XVII: 27-40. https://ojs. uv.es/index.php/qdfed/article/view/3440/3143

Martínez Gallego, Francesc. 2020. Paraules a batre. L’Alcúdia, 752: 22.

Martínez Mazaga, Uxune. 20I4. Emakumeak, zientzia eta diskriminazioa: Mateotik Matilda efektura. Zientzia Kaiera (blog) https://zientziakaiera.eus/20I4/10/03/ emakumeak-zientzia-eta-diskriminazioa-mateotik-matilda-efektura/

Mercier, Louis Sébastien. 2009. Néologie. Texto editado, anotado y presentado por Jean-Claude Bonnet. Paris: Belin. doi: I0.4000/ahrf.II83I

Merton, Robert K. 1968. The Matthew Effect in Science. Science vol. I59 (3810): 56-63. doi: I0.II26/science.I59.3810.56

Murcia, Sandra. 2020. Entrevista a Silvia Rueda. AlicantePlaza. https://alicanteplaza. es/silvia-rueda-en-el-mundo-stem-somos-pocas-y-en-puestos-de-responsabilidad-casi-desaparecemos 
Pozo-Sánchez, Begoña \& Padilla-Carmona, Carles. 2020. Criptogínia: una paraula nova per a un fenomen antic. Eldiario.es. https://www.eldiario.es/cv/ opinion/Criptoginia-paraula-nova-fenomen-antic_6_992560739.html [Acceso 05/02/2020]

Roca, Rafael. 2020. Criptogínia. Levante EMV. https://www.levante-emv.com/cultura /panorama/2020/II/25/criptoginia-25558360.html

Rositter, Margaret W. 1993. The Matthew Matilda Effect in Science. Social Studies of Science vol. 23 (2): 325-34I. doi: 10.1177/030631293023002004

Russ, Joana. 2019. Cómo acabar con la escritura de las mujeres. Madrid: Dos Bigotes.

Snyder, Benson R. I97I. The Hidden Curriculum. Boston: Alfred A. Knopf.

Zacarés, Amparo. 2020. Criptoginia. Levante EMV. https://www.levante-emv.com/ opinion/2020/I2/II/criptoginia-26I6II52.html 